Nervenarzt 2013 $\cdot 84: 1279-1280$

DOI 10.1007/s00115-013-3830-8

Online publiziert: 30. Oktober 2013

(c) Springer-Verlag Berlin Heidelberg 2013

\section{W. Maier}

Klinik für Psychiatrie und Psychotherapie der Universität Bonn

\title{
Von der Therapie zur Prävention
}

Das Motto des Kongresses der Deutschen Gesellschaft für Psychiatrie und Psychotherapie, Psychosomatik und Nervenheilkunde (DGPPN) 2013 „Von der Therapie zur Prävention" ist ein in der Medizin und im Gesundheitswesen allgemein anerkanntes Prinzip. Es gilt als ethisches Gebot und stellt eine gesetzliche Verpflichtung nach dem Sozialgesetzbuch (SGB) dar. Für psychische Erkrankungen war ein solches Programm bis vor kurzem kaum realisierbar. Bevölkerungsbezogene und krankheitsunspezifische Ansätze waren in der Vergangenheit bezüglich psychischer Erkrankungen nur wenig erfolgreich. In Forschung und Versorgungsmodellen wird derzeit dieser Entwicklungsstillstand überwunden. Diese Fortschritte beruhen auf krankheitsspezifischen Konzepten zu Ursachen und Entstehungsmechanismen, zu Pathogenese und Vorverlauf der Störungen und zu den möglichen Wirkmechanismen von Interventionen. In Versorgungsmodellen erweist sich die indizierte Prävention, die an krankheitsbezogenen Vorläufersymptomen (Prodromalzeichen) bzw. an krankheitsfördernden Risikofaktoren und Risikobedingungen (primäre Prävention) ansetzt, als richtungsweisend. Der diesjährige DGPPNKongress beleuchtet erfolgreiche Neuentwicklungen und Perspektiven in der primären und indizierten Prävention psychischer Störungen.

Indizierte Prävention: Zunehmend haben sich Methoden der Früherkennung und Frühintervention zu einem Schwerpunkt in der klinischen Forschung und in Modellprojekten entwickelt. Motivierend für diese Fortschritte war die Erkenntnis, dass viele psychische Krankheiten viel zu spät erkannt und behandelt werden und möglicherweise aus diesem Grund
Chronifizierungen begünstigen. Die Erkennung symptomatischer Frühwarnzeichen (Prodromi) noch vor der Manifestation des diagnostischen Vollbildes wurde durch jahrzehntelange, gezielte psychopathologische und epidemiologische Forschung ermöglicht. Hieraus entstanden Früherkennungs- und Interventionsprogramme, die derzeit an forschungsorientierten Standorten weltweit - v. a. in Bezug auf die Diagnosen Psychose/Schizophrenie, Demenzen und bipolare Störungen - in Entwicklung, Evaluation, teilweise auch im Prozess der Implementierung in das Versorgungssystem sind.

In dieser Kongressausgabe von Der Nervenarzt werden zwei eindrucksvolle Beispiele für indizierte Prävention dargestellt: die international mittlerweile in der klinischen Forschung erfolgreich etablierten und leider nur langsam in die Regelversorgung übergehenden Programme zur Frühintervention bei Psychosen/Schizophrenie (Beitrag J. Klosterkötter) sowie die noch in der ersten Entwicklungsphase steckende Früherkennung bei bipolaren Störungen (Beitrag „Prävention bipolarer Störungen“ von K. Leopold, A. Pfennig, E. Severus und M. Bauer).

Risikofaktorgestützte Primärprävention: Die Primärprävention setzt noch einen Schritt vor der indizierten, d. h. der auf Frühsymptome und -zeichen gestützten Prävention an. Ihre Zielpopulation sind Menschen ohne Krankheitssymptome (Beschwerden) oder -diagnosen. Primärprävention erfolgte bisher in einer relativ unspezifischen Weise ohne Bezug auf spezifische Störungen und deren Entstehungsmechanismen. Daher war die Wirksamkeit primärpräventiver Maßnahmen, von wenigen Ausnahmen abgesehen (z. B. Krebsprävention durch Antiraucherkam- pagnen), kaum erfolgreich. Die bisher nur unzulänglich berücksichtigte medizinische, krankheitsspezifische Expertise macht in Zukunft gezieltere und erfolgreichere Primärpräventionsprogramme in Populationen mit Risikofaktoren möglich. Ein klassischer Ort der Primärprävention ist der Arbeitsplatz. Das öffentliche und politische Interesse ist bei dieser Thematik besonders stark.

Betriebs- und Hausärzte sind ärztlicherseits bevorzugt mit Gesundheitsfolgen von Risikofaktoren konfrontiert. Dagegen findet die Primärprävention bisher kaum in der fachärztlichen Versorgung statt. Die Entwicklung effizienter störungsspezifischer Prävention (z. B. von Depression oder Angststörungen durch arbeitsplatzbezogene Risikofaktoren) erfordern aber krankheitsspezifische Kompetenz. Ein beispielhafter Ansatz - bezogen auf Risikofaktoren am Arbeitsplatz - ist im Beitrag von M. Berger et al. beschrieben.

Interventionsstrategien in Entwicklung: Bisherige Präventionsprogramme bei psychischen Störungen nutzen evidenzbasierte wirksame Therapien bei voll ausgeprägten psychischen Störungen, um sie auch präventiv bzw. frühzeitig einzusetzen (s. Beiträge J. Klosterkötter und $M$. Berger et al.). Bei der indizierten Prävention von Psychosen hat dieser Ansatz teilweise Kritik hervorgerufen angesichts des Nebenwirkungsprofils dieser Wirkstoffe und der damit verbundenen möglichen „Stigmatisierung“ von Betroffenen. Daher werden die evidenzbasierten psychotherapeutischen Interventionen zunehmend präventiv favorisiert und eingesetzt, ebenso wie nebenwirkungsarme, natürliche Wirkstoffe. Die präventiven Wirkmechanismen und die „biologische Plausibilität" 
solcher putativ wirksamen Interventionen müssen aber noch entschlüsselt werden, um Präventionsmaßnahmen zu optimieren. Zwei Beiträge in dieser Ausgabe sind dabei differenten Strategien gewidmet (Beitrag zu Oxytocin von M. Eckstein und R. Hurlemann sowie Beitrag zur modularen Psychotherapie von C. Schmahl und M. Bohus).

Ein aktueller historischer Bezug: Die zunehmende Bedeutung von Prävention rührt an Grundlagen unseres wissenschaftlichen und ethischen Grundverständnisses in unserem Fach. So treten Fragen zur Grenze zwischen gesund und krank und zur Natur psychischen Krankseins bzw. psychischer Krankheit wieder in den Vordergrund. Diskussionen zu diesen Themen durchziehen die Geschichte unseres Faches. Einen historischen Glanzpunkt stellt dabei die Monographie „Allgemeine Psychopathologie“ von Karl Jaspers dar, die vor 100 Jahren in erster Auflage erschien. Grundsätzliches zu diesem Werk und seiner Wirkung, zu dessen $\mathrm{Au}$ tor und seinem Denken ist im ersten Beitrag dieser Ausgabe von H. Häfner zu lesen.

In diesem Sinne wünsche ich Ihnen bei der Lektüre der folgenden Seiten ebenso wie auf unserem Kongress 2013 vielfältige Impulse und rege Diskussionen.

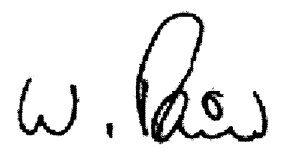

Prof. Dr. W. Maier

\section{Korrespondenzadresse}

\section{Prof. Dr. W. Maier}

Klinik für Psychiatrie und Psychotherapie

der Universität Bonn

Sigmund-Freud-Straße 25, 53127 Bonn

Wolfgang.Maier@ukb.uni-bonn.de

Interessenkonflikt. W. Maier gibt an, dass kein Interessenkonflikt besteht.

\section{T. Kircher (Hrsg.) \\ Kompendium der Psychotherapie}

Springer 2012, 454 S., (ISBN 978-3-642-23663-1), softcover, mit CD, 69.95 EUR

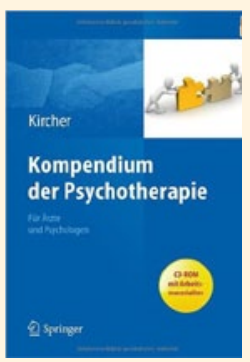

Dieses Mehrautoren-

buch der Marburger

Universitätsklinik für

Psychiatrie verfolgt

den Zweck, ärztliche und psychologische

Psychotherapie

als integralen Bestandteil unseres

psychiatrischen Fachgebietes kompetent und hochstrukturiert darzustellen. Es ist primär an junge Ärzte und Psychologen gerichtet, die sich entweder in der Weiterbildung zum Facharzt für Psychiatrie und Psychotherapie befinden oder in der Ausbildung zum psychologischen Psychotherapeuten und danach ihren Schwerpunkt im Bereich der Psychiatrie suchen. Alle Kapitel sind einheitlich strukturiert, es gibt sehr viele informative Darstellungen, sei es als Abbildung oder als Tabelle, und mit Spiegelstrichen wird das Wichtigste zusammengefasst, Fallbeispiele machen den theoretischen Stoff des Vorgehens sehr anschaulich.

Es ist im Grunde primär ein Buch mit „Kochrezepten" im besten Sinne des Wortes, mit denen noch nicht so in Psychotherapie Erfahrene für jedes Krankheitsbild ein klares Vorgehen in Modulen mit dem notwendigen theoretischen Rüstzeug vermittelt bekommen. Das ist für den klinischen Alltag ausgesprochen hilfreich, da die jungen Kollegen anhand dieses Materials ganz konkret an Patienten arbeiten und ihre ersten psychotherapeutischen Erfahrungen machen können. Darüber hinaus ist das Buch auch zum einen für diejenigen geeignet, die zwar schon länger dabei sind, aber psychotherapeutisch nur temporär arbeiten, aber auch für Angehörige anderer Berufsgruppen. Alle psychiatrischen Krankheitsbilder werden abgehandelt, insbesondere sind die Grundlagenkapitel am Anfang sehr zu empfehlen, in denen der Leser das grundsätzliche Vorgehen bei der Psychotherapie erfassen und erfahren kann. Ein wenig kritisch muss man anmerken, dass das Buch primär aus dem Blickwinkel der Verhaltenstherapie geschrieben ist und damit nicht als ein Kompendium der gesamten
Psychotherapie dienen kann, sondern eben speziell dieses Verfahrens, welches jedoch in der akuten Psychiatrie von großer Bedeutung ist. Zum anderen wäre ein größeres Kapitel zu Persönlichkeitsstörungen noch nützlich gewesen - so wird nur die Borderline-Persönlichkeitsstörung dargestellt -, da die AchseII-Störungen in der Psychiatrie eine große Rolle spielen. Das schmälert aber den Wert dieses innovativen Buches in keiner Weise. Es ist höchst verdienstvoll, gerade aus propädeutischen Gründen jungen Kollegen ein stringentes Buch über ungefähr 400 Seiten inklusive einer $C D$ an die Hand zu geben. Ich bin überzeugt, dass dieses Buch eine breite Leserschaft und Anwendung insbesondere in den psychiatrischen Kliniken finden wird. Des Weiteren wird es den Wert der Psychotherapie in unserem Fachgebiet stärken und- so hoffe ich - auch die ärztliche Psychotherapie und ihren Stellenwert verbessern.

G. Juckel (Bochum) 